\title{
How to Improve Clinical Research Performances in France?
}

\author{
Vincent Diebolt ${ }^{1}$, Yannick Pletan ${ }^{2}$ and the participants in Round Table $N^{\circ} 4$, Giens XXIII ${ }^{\star}$ \\ 1 GIP CeNGEPS, Direction Générale, Hospices Civils de Lyon, Lyon, France \\ 2 Pfizer, Paris, France
}

Text received 17 July 2008 ; accepted 30 July 2008

\section{Keywords:}

clinical research; feasability; qualification; efficiency

\begin{abstract}
The general objective of this study was to proceed an inventory of measures which could help to improve the efficiency of clinical research in France.

Method. Thanks to the discussion between the members of the round-table conference (composed of medical doctors (MD)/investigators; hospital managers; representatives of industrial promoters; general practitioners...), we have looked over the difficulties that meets clinical research in France nowadays.

Results. We identified four fields of action: 1) availibility of resources for carrying out the trials in time and in quality; 2) feasability of the clinical trials, in their design and for the recruitment which depends on both of the investigation centers and patients (what is the image of human testing in the society?); 3) skills and motivation of all the elements of staffs in charge of clinical trials (MD; nurses; and the new jobs in medical research); 4) promptness and efficiency of the administrative organisation for a fast starting up of the trials.

Conclusion. This study, which doesn't pretend to sufficiency, shows the gap of improvement for French clinical trials organisation.
\end{abstract}

From the outset, it seemed important to specify the meaning given to the term "performance" in the workshop. Indeed, we must distinguish the actual research objective and its results, making it possible to reach a scientific hypothesis, from its operational dimension, leading to the satisfaction of a certain number of internationally acknowledged criteria, which define France's level of appeal in comparison countries undertaking these same activities and in a context of international competitiveness. This roundtable focussed on the second dimension.

The first discussion then looked at identifying major guidelines for improving clinical research. Four factors emerged:

1. The availability of the resources needed to conduct research protocols (availability in quantity and on time in the shortest time limits).

2. The feasibility of the trial protocol design and recruitment depending on the centre/team/investigation network (recruitment commitment) and patients and the image they have of biomedical experimentation.

\footnotetext{
* For a list of participants, see the end of the article.
}

3. The qualifications and motivations of associated individuals and professions that comprise the team leading the research protocols.

4. The flexibility and efficiency of the administrative organisation with regards to the shortest preliminary trial preparation times.

For each of the factors conditioning trial fulfilment in scheduled time limits and expected quality standards, the working group has drawn up a certain number of recommendations enabling the current situation to be improved.

\section{Performing a true-up operation on dedicated and necessary resources (of all kinds)}

Today, determining the resources needed to conduct trials remains small-scale and approximate, without any real anticipation of the necessary production factors. As a result, the trials conducted are handicapped from the outset. 
Four proposals were selected by the roundtable participants.

1. Developing skills and tools for "evaluating the necessary means (time/costs)" for conducting clinical research projects, with the possibility of mutualising institution resources (nonteaching hospitals, for example) that as a consequence do not have the critical size or level of clinical trial activity.

2. Funding traceability and flagging: recognising operating services' clinical research without distinguishing the origin (whether its resources come from public/PHRC, or private funds/industrial promoted trials, etc.).

3. Harmonising the calculation of additional clinical trial hospital expenditure by drawing up a single grid for all hospital institutions. This general grid should include investigators fees (one-stop shop for pharmaceutical industries), as already exists in cancer centres.

4. ... and ensuring that research takings really do cover research activity expenditure..

\section{Guaranteeing "true" trial feasibility for fulfilment within the given deadline and on budget}

Nine different measures were considered by the roundtable members:

1. Ensuring the availability of dedicated professional logistical expertise in investigation centres.

2. Bringing together research staff and staff from the clinical research and innovation delegation (DRCI), rather than affectation to clinical departments, in order to ensure the mutualisation of means and affectations, for optimal use through building mobile pools.

3. Ensuring the visibility of the service offer of each clinical research entity (hospital; office-based practice; network, etc.).

4. Having recourse to networks. A number of innovative solutions have been quoted, enabling efficient "urban hospital" networks to be activated: soliciting general medical departments within medical faculties; relying on networks of exsenior registrars now set up in office practices...

5. Ensuring information and transparency regarding the performance of inclusion centres: developing a directory of centres detailing their scientific nature and inclusion capacity.

6. Taking into account the different targets depending on the nature of the trial:

- Depending on whether the trial focuses on chronic or rare pathologies, or acute episodes, the inclusion centres to be activated will not be the same. In the first case, reference centres and patient associations will have to be solicited; emergency services and office-based doctors for the second.

- Integral scientific and operational feasibilities: e.g. for socalled cluster or network trials on rare diseases, the recruitment plan should be adapted as a larger number of centres need to be included in order to recruit the planned number of patients, a number that can only be restricted. There is therefore a need to adapt the management of such scarcity.

7. Ensuring greater availability of the industry's personnel alongside operational teams, notably with a medical presence.

8. Developing tools for evaluating inclusion capacities:

- Use of PMSI and personal medical files (PMF) when they have been developed.

- Pooling of databases, registers and cohorts to identify "eligible" patients, possibly including those at the disposal of the health insurance sector, preserving the files' anonymity of course.

9. Appointing a national pharmaceutical public institution (perhaps the AGEPS at Paris Hospitals). This institution could centralise the healthcare institution preparation of clinical supplies (placebos) and preparations for therapeutic or associated trials.

\section{Staff professional development and motivation: Status}

\section{1. Training}

Three measures were proposed to emphasize the clinical research formation.

- Introduction to clinical research in initial training for the various health professions concerned (critical reading of articles in national qualification exams $(\mathrm{ECN})$; former entrance examinations, etc.).

- Inclusion into continued, qualifying or validating Accredited CME training. This could lead to the allocation of a "clinical research practitioner" title for the medical body.

- Qualifying or validating training (continuing medical education [CME]; evaluation of professional practices [EPP]; recognition of prior learning [RPL]; career acceleration (medical doctors, nurses).

\subsection{Recruitment}

Four actions should contribute to support the professionnalisation of clinical research activity.

- Taking into account experience in and knowledge of clinical research in the criteria/job descriptions of healthcare institution practitioners. 
- Obtaining authorisation from the investigator service sites based on the recognised level of staff training.

- Transparency and structuring of the profit-sharing scheme.

- Developing teamwork for publications in order to recognise the contribution of everyone in the writing of articles, not just that of the head of the research institution.

\subsection{Recognition and motivation}

Six actions were planned in order to develop the participation in clinical research activity.

- Boosting clinical research [CR] image (/personnel+/patients+general public): "CR is systematic"... National awareness and local communication actions.

- Attending steering committees, which entails good working knowledge of English.

- Restoring the balance of consideration for clinical research versus basic research when taking into account the evolution of investigator careers.

- Perpetuating clinical research professional positions proportionately with departments' effective "research" activity.

- Diversifying professional careers alongside recognition of clinical research training/specialisation (career development; grade-related bonuses, etc.).

- Developing Investigator Sponsored Studies (facilitate access to molecules and quality management).

\section{Simplifying administrative procedures for implementing and running research protocols}

Standard contracts in force in the entire country (saving time and human resources).

A single contract for any given institution (no longer an associated individual investigator agreement).

\section{Prospects}

Workshop participants suggested a number of "good reasons" for health industry players to come and work in France:

- A high added-value offer for early trials (phase I, proof of concept, translational research) around "Competitiveness Centres", with highly specialised staff, technical facilities and accessible, adapted biological resource centres. Healthcare "Competitiveness Clusters" present a major opportunity, since they ensure favourable territorial and administrative grouping.

- Far-reaching experience in later development phases, with tested and widespread practice of good clinical practice (GCP) and ethical practice (historically the Huriet Law). Network development, allowing for obligatory competition in reaching objectives for necessary staff numbers, is an essential shortterm step.

- France is the third pharmaceutical market in the world. This position encourages many international pharmaceutical groups to agree to potentially higher research costs in order to gain access to this market at an earlier stage. This is particularly true for certain therapeutic sectors (cardiovascular; CNS [central nervous system]; rheumatology).

- Specific expertise has been recognised in areas such as cancer, AIDS and infectious diseases (fungal infections; antibiotic therapy, etc.) explaining France's relative over-representation in these sectors $(30 / 40 \%$ of worldwide oncology developments are made on French soil).

- These assets are to be developed at international level. Initially, the quality and ease of implementing better expertise must be proven. At a later stage, one will have to accept a comparison of commonly recognised international "metrics" around notions of recruitment speed and intensity, reliability/quality of data and efficiency costs.

- The development approach should be proactive in relation to study designers. Pharmaceutical groups, "developers" must be contacted and convinced based on these criteria, bearing in mind that these criteria will evolve over time and the need to ensure that the offer's pertinence persists in a competitive environment (in order not to offer something that is obsolete).

\section{Issues not alluded during the workshop}

Some issues were not discussed during the workshop due to lack of time. From the point of view of the participants, the following should nevertheless be brought up:

- The role of the French national medical association (CNOM): bringing awareness (deontology; help with contract signing; conveys image of notoriety).

- BioTechs as emerging research sponsors, lacking the experience and resources of "major pharmaceutical players". The subject calls for an in-depth look at collaborative models (e.g. within the framework of competitiveness clusters); variable geometry partnerships, etc.

- Biobanks; collections representing a central element for tomorrow's trials (diagnostic; targeting; therapeutic solution; epidemiology; pharmaco-epidemiology).

- The role of CROs, as performance catalysts, and the contribution of expertise resources (logistics; skills; tools; methodology, etc.).

- The AERES and its role in developing efficient clinical research evaluation. 
At the end of the workshop, a list of five priority actions, actions which cannot wait, was produced... :

- Creating stable professional investigation teams in inclusion centres.

- Developing the image of clinical research and medical experimentation in the media and with patients.

- Harmonising technical-regulatory practices.

- Creating the post of trial "logistician" to anticipate and ensure the resolution of critical trial feasibility issues.

- Being pro-active by informing authorities and organisations and federating initiatives: with CSIS; CeNGEPS; CNCR (representing three conferences); Leem; CRBSP; DIRRC/DRCI; INPES, etc.

- SMO (site management organisation) experience used quasisystematically in the United States, working on behalf of pharmaceutical companies or institutional sponsors, allowing personnel and logistical means to be made available locally, directly facilitating the activity of investigation teams. The concept of "pure" SMOs has not yet emerged in France whereas the need is increasingly significant, with investigation teams being proportionally more restricted. Thus, some CROs have integrated certain SMO logistical functions, despite their different positioning.

- The public funding of clinical research projects (CRP, etc.), which feeds the sector and contributes to the professional development of investigators and research teams, whose continuity needs to be ensured.

- The overhead that ensures full financial coverage, including organisation and project management expenditure.

- Going beyond the borders of France to implement a European clinical research network from France through the implementation of identified investigation networks (example to follow: cardiology [Lille; Nancy]; CNS/geriatrics [Toulouse]).

\section{Abbreviations:}

AERES: Agence d'Évaluation de la Recherche et de l'Enseignement

AGEPS: Agence Générale des Équipements et Produits de Santé - General agency of medical devices and health products (pharmaceutical department of Public Assistance-Hospitals of Paris)

CeNGEPS: National Centre for management of healthcare product trails

CME: Continuing medical education

CNCR: Comité national de coordination de la recherche des CHU

- National comity for research activity of the french university hospitals

CNOM: Conseil national de l'Ordre des médecins - French medical National Association

CRBSP: Comité de la Recherche Biomédicale en santé publique - Comity of biomedical research in public health

CRO: Contract Research Organisation

CRP: Clinical research projects

CSIS: Comité Stratégique des industries de santé - Strategic comity of health's industries
DIRRC: Délégation inter-régionale à la recherche clinique - Interregional delegation of clinical research

DRCI: Délégation à la recherche clinique et à l'innovation - Delegation (or Department) of clinical research and innovation

ECN: Épreuves nationales classantes (ou examen national classant) - National exam for housemen

EPP: Évaluation des pratiques professionnelles (evaluation of professional practices)

INPES: Institut National de Prévention et d'Éducation à la santé Leem: Les Entreprises du Médicament - Pharma Industry Union PHRC: Projet hospitalier de recherche clinique

PMSI: Programme demédicalisation des systèmes d'information - Personal medical information system

PMF: Personal medical files

RPL: Recognition of prior learning

SMO: Site management organisation

\section{Participants:}

François Ballet (Sanofi Aventis), Jehan Michel Behier (MSD Chibret), Pierre Bey (Institut Curie, Paris), Antoine Cournot (Théraplix), Hervé Decousus (CHU Hôpital de Bellevue, Saint-Étienne), Jean Deregnaucourt (Pierre Fabre), Élisabeth Devilliers (Fédération Hospitalière de France [FHF]), Claude Dubray (CIC, Clermont Ferrand), Claude Gaultier (INSERM), François Gueyffier (Hôpital Cardiovasculaire Louis Pradel, Lyon Bron), Jean Michel Joubert (UCB Pharma), Rémi Le Coent (GlaxoSmithKline), Françoise Le Thiec (Janssen Cilag), Pascale Maisonneuve (Afssaps), Christine Marey (Science Union Les Laboratoires Servier), Pierre Marquet (Hôpital Dupuytren, Limoges), Dominique Menuet (Pfizer), Emmanuel Ogier (CIC, Brest), Atul Pathak (Pharmacologie Clinique, Toulouse), Éric Postaire (Direction Genérale de la Recherche et de l'Innovation Paris [DGRI]), Olivier Rascol (Faculté de Médecine, Toulouse), Jean Charles Reynier (AP-HM CHU, Marseille), Patrick Rossignol (CIC INSERM, Dommartin les Toul), Christian Thuillez (CHU, Rouen), Françoise Trinquet (AstraZeneca), Philippe Unger (Euraxipharma, Truyes).

Correspondence and offprints: Vincent Diebolt, GIP CeNGEPS, Direction Générale, Hospices Civils de Lyon, 3 quai des Célestins, 69229 Lyon Cedex 02, France.

E-mail: v.diebolt@cengeps.fr 